\title{
El papel de la inmunidad innata en la obesidad
}

\author{
Ángeles Fortis, M en C, ${ }^{(1,2)}$ Rebeca García-Macedo, D en C, ${ }^{(2)}$ Carmen Maldonado-Bernal, D en C, ${ }^{(3)}$
}

Francisco Alarcón-Aguilar, D en C, ${ }^{(3)}$ Miguel Cruz, D en C.(4)

\section{Fortis A, García-Macedo R, Maldonado-Bernal C, Alarcón-Aguilar F, Cruz M. El papel de la inmunidad innata en la obesidad. Salud Publica Mex 2012;54:17I-I77.}

\section{Resumen}

La obesidad en México es un problema de salud preocupante por el incremento en la prevalencia en adultos y niños, y se considera un factor de riesgo para el desarrollo de resistencia a la insulina, así como de otras alteraciones metabólicas. En esta patología se ha observado un incremento en la expresión de los receptores tipo Toll (TLRs) en el adipocito, receptores con participación crucial en la respuesta inmune innata. Se propone que los TLRs están implicados en la inflamación sistémica y en el desarrollo de la resistencia a la insulina. La activación de los TLRs es mediada por ácidos grasos y su expresión está regulada por leptina, adiponectina y PPAR. El conocimiento de la función de los TLRs, tanto en la inflamación como en la diferenciación del adipocito es importante en la búsqueda de nuevos blancos terapéuticos antiinflamatorios que coadyuven en el tratamiento de la obesidad.

Palabras clave: receptores tipo Toll; obesidad; inflamación; resistencia a la insulina

\author{
Fortis A, García-Macedo R, Maldonado-Bernal C, \\ Alarcón-Aguilar F, Cruz M. \\ The role of innate immunity in obesity. \\ Salud Publica Mex 20I2;54:17I-I77.
}

\section{Abstract}

Obesity in Mexico is alarmingly increasing in prevalence in adults and children, and it is a risk factor for the development of insulin resistance, as well as, of other metabolic alterations. The discovery of the expression of the Toll-like receptors (TLRs) in adipocytes, suggests an important role in innate immunity. In different models of obesity, there has been observed an increase of TLRs expression in the fat tissue, therefore TLRs could be involved in systemic inflammation in this disease, and in the development of insulin resistance. TLR activation is mediated by fatty acids and their expression is regulated by leptin, adiponectin and PPARs. Knowledge of the role of TLRs in inflammation and adipocyte differentiation and their regulation, then it is important to try to develop new therapeutic anti-inflammatory targets that contribute in the treatment of obesity.

Key words: toll-like receptor; obesity; inflammation; insulin resistance

(I) Programa de Doctorado en Biología Experimental, Universidad Autónoma Metropolitama. México DF, México.

(2) Unidad de Investigación Médica en Bioquímica, Hospital de Especialidades, Centro Médico Nacional Siglo XXI, Instituto Mexicano del Seguro Social. México DF, México

(3) Unidad de Investigación en Enfermedades Oncológicas, Hospital Infantil de México Federico Gómez, Secretaría de Salud. México DF, México

(4) Departamento de Ciencias de la Salud. División de Ciencias Biológicas y de la Salud. Universidad Autónoma Metropolitana. México DF, México

Fecha de recibido: 6 de abril de 20II - Fecha de aceptado: 22 de noviembre de 20II

Autor de correspondencia: Dr. Miguel Cruz. Unidad de Investigación Médica en Bioquímica, Hospital de Especialidades, Centro Médico Nacional Siglo XXI, Instituto Mexicano del Seguro Social. Av. Cuauhtémoc 330, Col. Doctores. 06720 México DF, México.

Correo electrónico: mcruzl@yahoo.com 
L a obesidad es un problema de salud pública crecienـte en los ámbitos mundial y nacional. En México, la incidencia y la prevalencia han aumentado de manera alarmante en los últimos 20 años; en hombres adultos se incrementó de 60 a 70\% entre los años 2000 a 2006, tasa de incremento que fue ligeramente inferior en mujeres. Además es preocupante el incremento del sobrepeso y obesidad en niños pues en 1999 la prevalencia fue de alrededor de $18.6 \%$ y en 2006 de $26 \% .{ }^{1}$ Se debe tener atención en este sector de la población ya que la obesidad es una patología que tiene implicaciones importantes en la generación de resistencia a la insulina y es un factor de riesgo para el desarrollo de la diabetes tipo 2 (DT2).

En la obesidad, el exceso de tejido adiposo blanco genera una respuesta inflamatoria crónica de bajo grado, debido a que este tejido incrementa la secreción de moléculas inflamatorias, como la leptina, el factor de necrosis tumoral alfa (TNF- $\alpha$ ), la interleucina (IL) 6 y la resistina, y se encuentra disminuida la secreción de la adiponectina, citocina con acción antiinflamatoria., ${ }^{2,3}$ Actualmente se reconoce que el tejido adiposo tiene implicaciones en la inmunidad innata debido a que es capaz de responder a agentes patógenos a través de sus receptores tipo Toll (TLRs). Se ha propuesto que la activación de los TLRs en el adipocito puede contribuir a la inflamación presente en la obesidad. ${ }^{4-7}$ Estos receptores favorecen la activación del factor nuclear-kappa B (NF-кB), que regula la transcripción de moléculas inflamatorias. ${ }^{8}$ Por su parte, las citocinas inflamatorias pueden desencadenar defectos en la transducción de señales de la insulina. Así, por ejemplo, el TNF- $\alpha$ produce resistencia a la insulina al inducir la fosforilación del sustrato del receptor de insulina (IRS) en residuos de serina y treonina, mediada por la cinasa $\mathrm{N}$-terminal de c-Jun (JNK) ${ }^{9,10}$ y la proteína cinasa IKK- $\beta{ }^{11}$ mientras que la IL-6 inhibe la fosforilación de IRS-1 e IRS-2 a través de la proteína supresora de la señalización por citocinas (SOCS-3). ${ }^{10,12}$ Por lo tanto, el estudio de los TLRs en el adipocito representa una interesante perspectiva para conocer nuevos mecanismos que podrían contribuir a la generación de la inflamación en la obesidad.

\section{LOS TLR}

Los TLRs son receptores que tienen una función importante en la inmunidad innata, por activación de señales proinflamatorias en respuesta a patógenos microbianos y ligandos endógenos. ${ }^{3,8}$ Son expresados en diversos tipos celulares, como macrófagos, células B y células dendríticas; ${ }^{8}$ recientemente se ha reconocido su expresión en los adipocitos. ${ }^{5,6}$ Se ha propuesto a los ácidos grasos saturados como ligandos endógenos de los TLRs en adipocito y se han asociado con la resistencia a la insulina ${ }^{5}$ (más adelante se abordará este tema en detalle).

Los TLRs activan dos vías: una dependiente del factor de diferenciación mieloide 88 (MyD88), activada por los TRL 1, 2, 4, 5, 6, 7, 8, 9 y 11, y la otra vía independiente de esta molécula, activada por el TLR3 y el TLR4. En la vía dependiente de MyD88, los TLRs activan a proteínas como a la cinasa asociada con los receptores de interleucina (IRAK) y al factor 6 asociado con el receptor del TNF- $\alpha$ (TRAF6). Consecutivamente, esta vía conduce a la activación del NF- $\kappa$ B y del activador de proteína-1 (AP-1), factores de transcripción que intervienen en la expresión de las citocinas inflamatorias. ${ }^{3,13}$ Esto causaría un aumento de la inflamación sistémica presente en la obesidad y como consecuencia en la generación de resistencia a la insulina. ${ }^{2,3}$ En la actualidad, aún falta por describir totalmente la cascada de señalización al activar cada uno de los TLRs expresados en el adipocito.

\section{Implicación de los TLR en la resistencia a la insulina}

Los ratones $o b / o b$ y $d b / d b$ presentan obesidad por defectos en la expresión de leptina y su receptor, respectivamente. En estos modelos se encuentra incrementada la expresión del TLR 1 al 9, tanto en preadipocitos como en adipocitos. Esto se ha asociado con alteraciones en la vía de señalización fosfatidilinositol 3-cinasa (PI3K), sugiriendo que la sobreexpresión de los TLRs puede afectar la transducción de señales de la insulina. ${ }^{5}$ Además, en los ratones ob/ob se encuentra alterada la respuesta inmune, observándose deficiencias en la respuesta a la infección por Klebsiella pneumonia ${ }^{14}$ y al tratamiento con zimozán (ligando del TLR2) para la inducción de artritis. ${ }^{15}$ Por lo tanto, la leptina, molécula mayoritariamente secretada por el adipocito, interviene en la respuesta del sistema inmunológico y en la señalización de la insulina a través de su influencia en la expresión de los TLRs.

La resistina es una citocina inflamatoria involucrada en la resistencia a la insulina; en adipocitos humanos incrementa la expresión de componentes del sistema inmune innato, entre ellos el TLR2, MyD88 y el NF-кB. ${ }^{16}$ Recientemente se ha propuesto que el mecanismo por el cual la resistina genera resistencia a la insulina es por la activación directa del TLR4, al unirse a este receptor. ${ }^{17}$

La obesidad es un factor importante para el desarrollo de la DT2. Esta patología se caracteriza por la presencia de hiperglucemia debida a defectos en la acción o secreción de insulina. ${ }^{18}$ En pacientes con DT2 
se ha encontrado aumento en la expresión del TLR2, así como concentraciones altas de insulina en plasma y resistencia a la insulina. ${ }^{19}$ En monocitos la hiperglucemia incrementa la expresión del TLR2 y del TLR4, a través de activación de la proteína cinasa C (PKC) y de la NADPH oxidasa. ${ }^{20}$ En otros casos, la insulina es capaz de suprimir la expresión del RNAm de los TLRs 1, 2, 4, 7 y 9 en células mononucleares, al inhibir la actividad del factor de transcripción PU1. ${ }^{21}$ La insulina es un regulador importante para la expresión de los TLRs, y sería interesante estudiar esta función en tipos celulares involucrados en el proceso inflamatorio de la obesidad.

Los ratones $\mathrm{C} 3 \mathrm{H} / \mathrm{HeJ}$ presentan disminución en la función de TRL4 y son resistentes a la inducción de obesidad por dieta alta en ácidos grasos en comparación con los ratones silvestres que presentan un aumento en la adiposidad, con incremento en la actividad de IKK- $\beta$ y JNK, presentando resistencia a la insulina en tejido adiposo y músculo. ${ }^{6}$ En el músculo de sujetos obesos con DT2 se encuentra altamente expresado el TLR4, con una asociación directa con la resistencia a la insulina y con la actividad del NF-kB. ${ }^{22}$ El efecto de mutar el TLR4 o el TLR2 produce una respuesta de protección a la obesidad y resistencia a la insulina, ${ }^{6,23}$ lo que ha generado gran interés por los TLRs como blancos terapéuticos en la obesidad.

\section{Activación de los TLR por ácidos grasos saturados}

En la obesidad se incrementa la infiltración de macrófagos al tejido adiposo y se correlaciona positivamente con el aumento en el peso corporal. ${ }^{10}$ Estos macrófagos secretan una gran cantidad de moléculas inflamatorias como el TNF- $\alpha$, molécula capaz de activar a los adipocitos para inducir la secreción de citocinas inflamatorias a través de la activación del NF-кB. También se ha determinado que pueden influir en el aumento de la lipólisis ${ }^{24}$ debido a la activación de la vía de las proteínas cinasas activadas por mitógenos (MAPK) que estimulan a la lipasa sensible a hormonas, ${ }^{10,24}$ lo que provoca la activación de los TLRs tanto en los macrófagos ${ }^{25,26}$ como en los adipocitos, ${ }^{2,25}$ lo que contribuye a generar la inflamación sistémica y la resistencia a la insulina en el propio adipocito ${ }^{4,13,27}$ por medio de mecanismos que disminuyen la activación del IRS por efecto del TNF- $\alpha$ e IL-6 (figura 1). ${ }^{9-12}$

En adipocitos 3T3-L1 el palmitato, el lipopolisacárido (LPS) y el zimozán inducen resistencia a la insulina por incremento en la expresión de IL-6 debido a la activación del NF-кB y del AP-1. ${ }^{27}$ Se sugiere que los ácidos grasos pueden ocasionar la resistencia a la insulina a través de la vía dependiente de MyD88 que activa el proceso inflamatorio. ${ }^{13}$ Además, esta vía podría activar

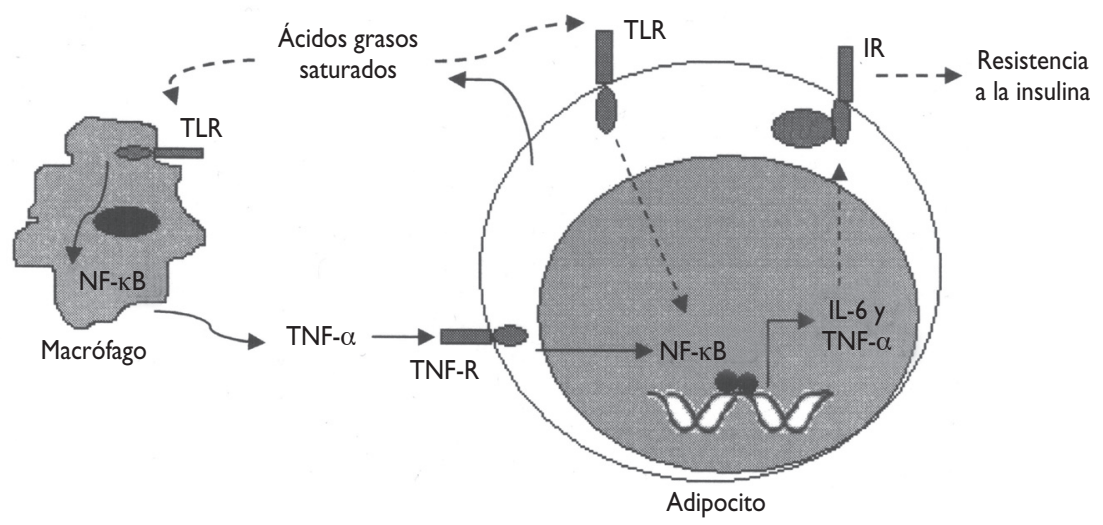

- - - Indican la vía de señalización que se propone para la activación de los TLRs

Figura I. Efecto de los ácidos grasos saturados en el adipocito y en el macrófago. Durante La iNfiLtración de LOS MACRÓFAGOS AL TEJIDO ADIPOSO SE PRODUCE UN AUMENTO EN LA SECRECIÓN DEL TNF- $\alpha$; ESTE FACTOR ACTIVA TANTO A LA LIPólisis COMO al NF-KB A tRAVÉs de SU ReCEPTOR (TNF-R) QUE SE ENCUENTRA EN EL ADIPOCITO. POR OtRO LADO, LOS ÁCIDOS GRASOS SATURADOS, PRODUCIDOS POR EL ADIPOCITO, ESTÁN INVOLUCRADOS EN LA ACTIVACIÓN DE LOS TLRS EN EL MACRÓFAGO Y EN EL PROPIO ADIPOCITO; ESTOS RECEPTORES TAMBIÉN FAVORECE LA ACTIVACIÓN DEL NF-KB, LO CUAL CONTRIBUYE A INCREMENTAR LA SECRECIÓN DE CITOCINAS INFLAMATORIAS EN ESTAS DOS ESTIRPES CELULARES. COMO CONSECUENCIA DISMINUYE LA ACTIVACIÓN DEL SUSTRATO DEL RECEPTOR DE INSULINA (IRS), CAUSANDO RESISTENCIA A LA INSULINA EN EL ADIPOCITO 
a cinasas (PKC y JNK) que fosforilan al IRS en el sitio de serina, disminuyendo los efectos de la insulina. ${ }^{4}$

Se ha determinado una alta expresión del RNAm del TLR4 en el tejido adiposo de ratones con obesidad generada por una dieta alta en grasas, así como en ratones $o b / o b$ y $d b / d b .{ }^{7}$ En ratones knockout para el TLR4 tratados con ácidos grasos saturados y LPS no se produce la activación del NF-kB, disminuyendo la expresión de citocinas proinflamatorias y, consecuentemente, la resistencia a la insulina. Además, en estudios in vitro usando RNA de interferencia específicos para bloquear la expresión del TLR4, se ha logrado corroborar que los ácidos grasos libres provocan una respuesta inflamatoria en adipocitos, observándose disminución en la expresión de citocinas inflamatorias (IL-6 y TNF- $\alpha$ ). 7,28

Diversos trabajos indican que el TLR4 es activado por los ácidos grasos; también se conoce que estos ácidos grasos inducen la expresión del TLR2 en tejido adiposo de ratón y en células 3T3-L1. ${ }^{29}$ En adipocitos 3T3-L1 se ha observado que la activación del TLR4 por LPS estimula la expresión del TLR ${ }^{29}$ el cual, a su vez, induce la expresión de citocinas proinflamatorias. ${ }^{30}$

Por otra parte, como ya se mencionó, se propone que los ácidos grasos son los ligandos directos de los TLRs, pero aún esta propuesta se encuentra en discusión, ya que algunos autores indican la existencia de ligandos intermediarios para la activación de los TLRs. ${ }^{31}$

Estos informes indican que una dieta alta en ácido grasos contribuye a la generación de la respuesta inflamatoria, causada por la activación de los TLRs, tanto en adipocitos como en macrófagos. Aunque ya se tienen antecedentes de los TLRs en la resistencia a la insulina, la mayor parte de estos estudios se enfocan en el TLR2 y el TLR4, pero no hay que olvidar que los adipocitos expresan otros tipos de TLR y aún no se conoce su función o si éstos intervienen en la inducción de la resistencia a la insulina.

\section{Participación de la adiponectina y de los receptores activados por proliferadores de peroxisomas (PPAR) en la expresión de los TLR}

La adiponectina es una citocina secretada por los adipocitos con actividad antiinflamatoria. En individuos obesos se observan concentraciones bajas de esta citocina, ${ }^{32}$ condición que se ha asociado con infecciones frecuentes. En macrófagos la activación del receptor AdipoR1 por la adiponectina inhibe la activación del NF- $\kappa B$ inducida por el TLR2 y el TLR4. ${ }^{33}$ Por otro lado, en adipocitos 3T3-L1 estimulados por peptidoglicanos la expresión del TLR2 aumenta, observándose disminución en la expresión de los receptores de la adiponectina, AdipoR1 y AdipoR2, lo cual favorece la inflamación presente en la obesidad. ${ }^{34}$ Así, la adiponectina podría tener una función importante en la regulación de las infecciones bacterianas de sujetos obesos. ${ }^{33}$

Se ha determinado que el PPAR- $\gamma$ tiene la capacidad de reprimir la expresión de los TLRs. ${ }^{35}$ En ratones $d b / d b$ el tratamiento con pioglitazona, un agonista del PPAR- $\gamma$, disminuye la expresión del TLR2 y del TLR4 en macrófagos, así como la activación del NF-KB. ${ }^{36}$ Las ratas Zucker, que presentan obesidad y diabetes, presentan disminución en la expresión y en la actividad de PPAR- $\beta$ y $-\delta$ en el tejido adiposo. Por otra parte, en adipocitos 3T3-L1, el estímulo con LPS activa la vía ERK1/ 2 y el tratamiento con un agonista del PPAR- $\beta$ y $-\delta$ inhibe la activación de esta vía, efecto que evita la activación del NF-kB y la secreción de citocinas proinflamatorias. ${ }^{37}$ Esto sugiere que los PPAR pueden inhibir la activación de los TLRs, así como la inflamación provocada por el tejido adiposo en la obesidad.

En general, la expresión de los TLRs en los adipocitos puede estar modulada por adipocinas como leptina y resistina, mientras que su activación puede estar mediada directamente por ácidos grasos o por la resistina, los cuales desencadenan vías de transducción de señales que activan al NF-kB, factor de transcripción que favorece la producción de citocinas proinflamatorias, disminuyendo la expresión de los receptores de la adiponectina y provocando resistencia a la insulina. Por otro lado, la activación de PPAR- $\gamma,-\beta$, - $\delta$ y la estimulación de la expresión de la adiponectina, inhiben la expresión de los TLRs y la activación del NF-kB (figura 2).

Además de su importancia en la inflamación, se ha determinado que los TLRs pueden estar involucrados en otros procesos fisiológicos.

\section{Participación de los TLR en la diferenciación de los adipocitos}

Durante la diferenciación de los adipocitos 3T3-F442A se encontró la coexpresión y la colocalización del TLR9 e IFN- $\gamma$. Se propone que el TLR9 tiene una función importante durante la diferenciación de los adipocitos por efecto de IFN- $\gamma \cdot{ }^{38}$ El mecanismo puede ser similar al presentado en células asesinas naturales (NK) de células dendríticas, en las cuales se ha comprobado que el IFN- $\gamma$ es capaz de activar al TLR9. ${ }^{39}$

Por otra parte, la activación del TLR2 en células mesenquimales por Pam3Cys inhibe su diferenciación a adipocitos; ${ }^{40}$ este efecto también ha sido observado en 


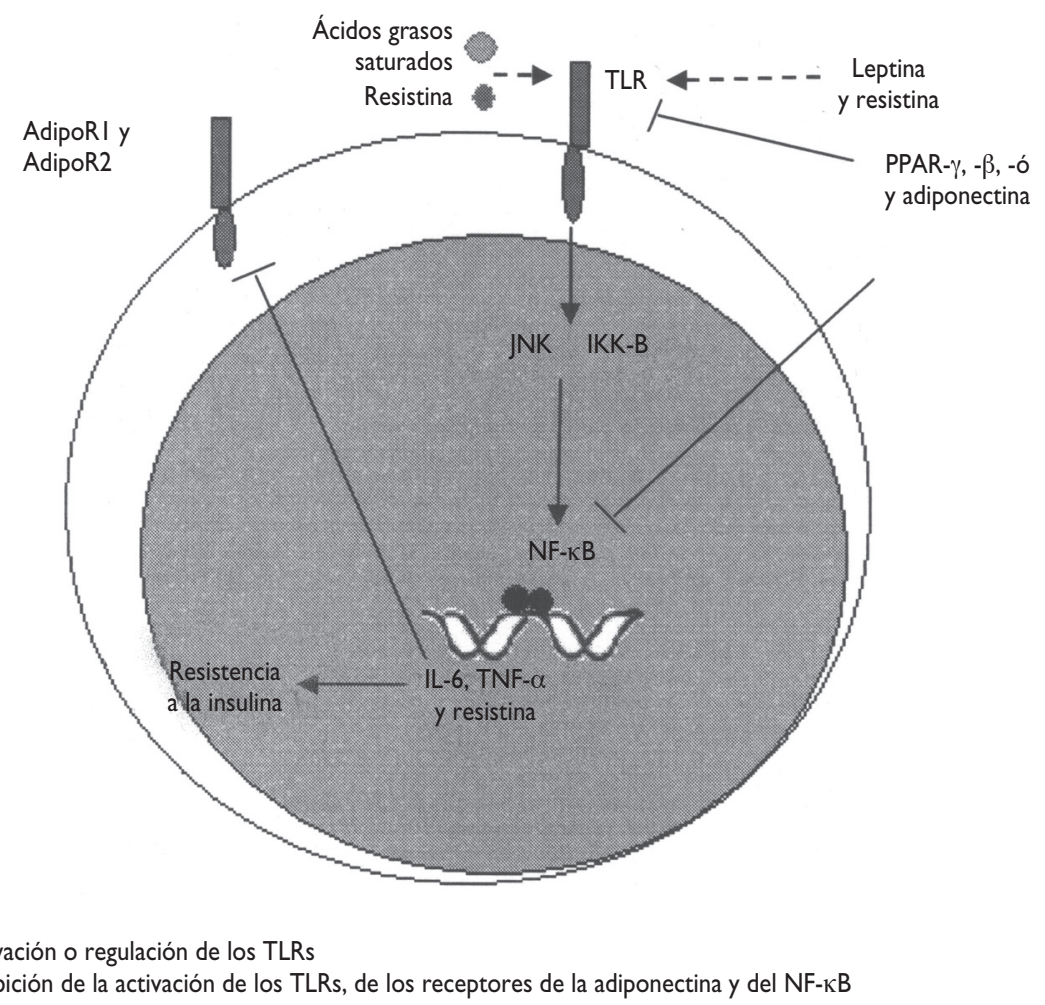

Figura 2. Efecto de Los TLRs SOBRe LA INFLAMACIÓN Y EL DESARROLLO de RESISTENCIA A LA INSULINA EN EL ADIPOCITO. LAS MOLÉCULAS SECRETADAS POR EL ADIPOCITO, COMO LEPTINA O RESISTINA, FAVORECEN LA EXPRESIÓN DE LOS TLRS, MIENTRAS QUE LA ADIPONECTINA Y LOS FACTORES DE TRANSCRIPCIÓN PPAR INHIBEN LA EXPRESIÓN DE LOS TLRS Y DEL NF-KB. Al activarse los TLRs POR LA RESISTINA O los ÁCIDOS GRASOS EN EL ADIPOCITO, SE ACTIVA NF-KB POR MEDIO DE CINASAS, TALES COMO JNK E IKK-B, LO CUAL CONTRIBUYE A LA GENERACIÓN DE LA INFLAMACIÓN, LO QUE DISMINUYE LOS RECEPTORES DE ADIPONECTINA E INCREMENTA LA RESISTENCIA A LA INSULINA

preadipocitos 3T3-L1 $1^{30}$ y la activación del TLR4 por LPS en preadipocitos inhibe la adipogénesis, disminuyendo la expresión de las enzimas de la lipogénesis y de citocinas tales como adiponectina, resistina y leptina, ${ }^{41}$ así como la activación y expresión del PPAR- $\gamma$, marcador de diferenciación celular. ${ }^{41,42}$

\section{Conclusiones}

Debido a que el estudio del adipocito se ha ligado principalmente al metabolismo de lípidos y carbohidratos, actualmente se conoce muy poco acerca de la participación del tejido adiposo en la inmunidad innata. Así por ejemplo, los ligandos responsables de la activación de los TLRs y las vías de señalización en el adipocito aún se desconocen. Los pocos estudios realizados al respecto indican que en el adipocito los TLRs son capaces de modular la respuesta inflamatoria a través del NF-KB y PPAR, con la participación de los ácidos grasos libres.
Esto abre una amplia gama de interrogantes en torno a los mecanismos implicados en el proceso inflamatorio mediado por el adipocito. Un mayor entendimiento de las funciones de los TLRs en el adipocito, tanto en la inflamación crónica como en la diferenciación celular, podría llevar al descubrimiento de nuevos blancos terapéuticos antiinflamatorios para el tratamiento de la obesidad y de las enfermedades crónico-degenerativas asociadas.

Declaration of conflict of interests: The authors declare that they have no conflict of interests.

\section{Referencias}

I. Olaiz-Fernández G, Rivera-Dommarco J, Shamah-Levy T, Rojas R, Villalpando-Hernández S, Hernández-Avila M, et al. Encuesta Nacional de Salud y Nutrición 2006. Cuernavaca, México: Instituto Nacional de Salud Pública, 2006. 
2. Franchini M, Monnais E, Seboek D, Radimerski T, Zini E, Kaufmann K, et al. Insulin resistance and increased lipolysis in bone marrow derived adipocytes stimulated with agonists of Toll-like receptors. Horm Metab Res 2010;42:703-709.

3. Fresno M, Alvarez R, Cuesta N. Toll-like receptors, inflammation, metabolism and obesity. Arch Physiol Biochem 201 I; I 7:I5I-I64.

4. Kim JJ, Sears DD. TLR4 and Insulin Resistance. Gastroenterol Res Pract 20I0: I-II

5. Batra A, Pietsch J, Fedke I, Glauben R, Okur B, Stroh T, et al. Leptin-dependent toll-like receptor expression and responsiveness in preadipocytes and adipocytes. Am J Pathol 2007; I70: 1931-194I. 6. Tsukumo DM, Carvalho-Filho MA, Carvalheira JB, Prada PO, Hirabara SM, Schenka AA, et al. Loss of function mutation in toll-like receptor 4 prevents diet induced obesity and insulin resistance. Diabetes 2007:56:1986-1998.

7. Shi H, Kokoeva MV, Inouye K, Tzameli I, Yin H, Flier JS. TLR4 links innate immunity and fatty acid-induced insulin resistance. J Clin Invest 2006; II 6:30I5-3025.

8. Carmody RJ, Chen YH. Nuclear factor-kappa B: activation and regulation during toll-like receptor signaling. Cell Mol Immunol 2007;4:3 I-4I.

9. Fernández-Veledo S, Vila-Bedmar R, Nieto-Vazquez I, Lorenzo M. c-Jun $\mathrm{N}$-terminal kinase I/2 activation by tumor necrosis factor-alpha induces insulin resistance in human visceral but not subcutaneous adipocytes: reversal by liver $\mathrm{X}$ receptor agonists. J Clin Endocrinol Metab 2009:94:3583-3593.

10. Torres-Leal FL, Fonseca-Alaniz MH, Rogero MM, Tirapegui J. The role of inflamed adipose tissue in the insulin resistance. Cell Biochem Funct 2010;28:623-631.

II. Gao Z, Hwang D, Bataille F, Lefevre M, York D, Quon MJ, et al. Serine phosphorylation of insulin receptor substrate I by inhibitor kappa B kinase complex. J Biol Chem 2002;277:48II5-48I2I.

I2. Senn J], Klover PJ, Nowak IA, Zimmers TA, Koniaris LG, Furlanetto RW, et al. Suppressor of cytokine signaling-3 (SOCS-3), a potential mediator of interleukin-6 dependent insulin resistance in hepatocytes. J Biol Chem 2003;278:13740-I3746.

13. Kopp A, Buechler C, Bala M, Neumeier M, Schölmerich J, Schäffler A. Toll-like receptor ligands cause proinflammatory and prodiabetic activation of adipocytes via phosphorylation of extracellular signalregulated kinase and c-Jun $\mathrm{N}$-terminal kinase but not interferon regulatory factor-3. Endocrinology 2010;151:1097-10108. 14. Mancuso P, Gottschalk A, Phare SM, Peters-Golden M, Lukacs NW, Huffnagle GB. Leptin-deficient mice exhibit impaired host defense in gram-negative pneumonia. J Immunol 2002; 168:4018-4024.

I5. Bernotiene E, Palmer G, Talabot-Ayer D, Szalay-Quinodoz I, Aubert ML, Gabay C. Delayed resolution of acute inflammation during zymosan-induced arthritis in leptin-deficient mice. Arthritis Res Ther 2004;6:R256-263. 16. Kusminski CM, da Silva NF, Creely SJ, Fisher FM, Harte AL, Baker $A R$, et al. The in vitro effects of resistin on the innate immune signaling pathway in isolated human subcutaneous adipocytes. J Clin Endocrinol Metab 2007;92:270-276.

17. Tarkowski A, Bjersing J, Shestakov A, Bokarewa MI. Resistin competes with lipopolysaccharide for binding to toll-like receptor 4. J Cell Mol Med 2010;14:1419-1431.

18. American Diabetes Association. Diagnosis and classification of diabetes mellitus. Diabetes Care 2004;27:SI-I0.

19. Creely SJ, McTernan PG, Kusminski CM, Fisher M, Da Silva NF, Khanolkar M, et al. Lipopolysaccharide activates an innate immune system response in human adipose tissue in obesity and type 2 diabetes. Am J Physiol Endocrinol Metab 2007;292:E740-747.

20. Dasu MR, Devaraj S, Zhao L, Hwang DH, Jialal I. High glucose induces toll-like receptor expression in human monocytes. Diabetes 2008:57:3090-3098.
2I. Ghanim H, Mohanty P, Deopurkar R, Sia CL, Korzeniewski K, Abuaysheh $\mathrm{S}$, et al. Acute modulation of toll-Like receptors by insulin. Diabetes Care 2008;31:1827-I83I.

22. Reyna SM, Ghosh S, Tantiwong P, Meka CS, Eagan P, Jenkinson CP, et al. Elevated toll-Like receptor 4 expression and signaling in muscle from insulin-resistant subjects. Diabetes 2008;57:2595-2602.

23. Himes RW, Smith CW. TIr2 is critical for diet-induced metabolic syndrome in a murine model. FASEB J 2010;24:731-739.

24. Permana PA, Zhang W, Wabitsch M, Fischer-Posovszky P, Duckworth WC, Reaven PD. Pioglitazone reduces inflammatory responses of human adipocytes to factors secreted by monocytes/macrophages. Am J Physiol Endocrinol Metab 2009;296:EI076-I084.

25. Schwartz EA, Zhang WY, Karnik SK, Borwege S, Anand VR, Laine

$P S$, et al. Nutrient modification of the innate immune response: a nove mechanism by which saturated fatty acids greatly amplify monocyte inflammation. Arterioscler Thromb Vasc Biol 2010;30:802-808. 26. Yamashita A, Soga Y, Iwamoto Y, Asano T, Li Y, Abiko Y, et al. DNA microarray analyses of genes expressed differentially in 3T3-LI adipocytes co-cultured with murine macrophage cell line RAW264.7 in the presence of the toll-like receptor 4 ligand bacterial endotoxin. Int J Obes (Lond) 2008;32:1725-1729.

27. Davis JE, Gabler NK, Walker-Daniels J, Spurlock ME. The c-Jun $\mathrm{N}$-terminal kinase mediates the induction of oxidative stress and insulin resistance by palmitate and toll-like receptor 2 and 4 ligands in 3T3-LI adipocytes. Horm Metab Res 2009;41:523-530.

28. Raetzsch CF, Brooks NL, Alderman JM, Moore KS, Hosick PA, Klebanov $S$, et al. Lipopolysaccharide inhibition of glucose production through the toll-like receptor-4, myeloid differentiation factor 88 , and nuclear factor kappa B pathway. Hepatology 2009;50:592-600. 29. Murakami k, Bujo H, Unoki H, Saito Y. High fat intake induces a population of adipocytes to co-express TLR2 and TNF alpha in mice with insulin resistance. Biochem Biophys Res Commun 2007;354:727-734. 29. Lin Y, Lee H, Berg AH, Lisanti MP, Shapiro L, Scherer PE. The lipopolysaccharide-activated toll-like receptor (TLR)-4 induces synthesis of the closely related receptor TLR-2 in adipocytes. J Biol Chem 2000;275:24255-24263.

30. Poulain-Godefroy O, Le Bacquer O, Plancq P, Lecoeur C, Pattou F, Frühbeck G, et al. Inflammatory role of Toll-like receptors in human and murine adipose tissue. Mediators Inflamm 2010;2010:823486.

31. Schaeffler A, Gross P, Buettner R, Bollheimer C, Buechler C Neumeier M, et al. Fatty acid-induced induction of Toll-like receptor-4/ nuclear factor-kappa B pathway in adipocytes links nutritional signalling with innate immunity. Immunology 2009; 126:233-245.

32. Rabe K, Lehrke M, Parhofer KG, Broedl UC. Adipokines and insulin resistance. Mol Med 2008;|4:74|-75|.

33. Yamaguchi N, Argueta JG, Masuhiro Y, Kagishita M, Nonaka K, Saito $\mathrm{T}$, et al. Adiponectin inhibits toll-like receptor family-induced signaling. FEBS Lett 2005;579:6821-6826.

34. Ajuwon KM, Banz W, Winters TA. Stimulation with peptidoglycan induces interleukin 6 and TLR2 expression and a concomitant downregulation of expression of adiponectin receptors I and 2 in 3T3-LI adipocytes. J Inflamm (Lond) 2009;6:8.

35. Ogawa S, Lozach J, Benner C, Pascual G, Tangirala RK, Westin S, et al. Molecular determinants of crosstalk between nuclear receptors and toll-like receptors. Cell 2005; I22:707-72I.

36. Dasu MR, Park S, Devaraj S, Jialal I. Pioglitazone inhibits toll-like receptor expression and activity in human monocytes and $\mathrm{db} / \mathrm{db}$ mice. Endocrinology 2009; 150:3457-3464.

37. Rodríguez-Calvo R, Serrano L, Coll T, Moullan N, Sánchez RM, Merlos M, et al. Activation of peroxisome proliferator-activated receptor beta/delta inhibits lipopolysaccharide-induced cytokine production in adipocytes by lowering nuclear factor-kappa B activity via extracellular signal-related kinase 1/2. Diabetes 2008;57:2149-2। 57. 
38. Khazen W, M'Bika JP, Collinet M, Tramoni M, Chany C, Achour A, et al. Differentiation-dependent expression of interferon gamma and toll-like receptor 9 in 3T3-F442A adipocytes. Biochimie 2007;89:669-675.

39. Chaudhry UI, Kingham TP, Plitas G, Katz SC, Raab JR, DeMatteo

RP. Combined stimulation with interleukin- 18 and $C P G$ induces murine natural killer dendritic cells to produce IFN-gamma and inhibit tumor growth. Cancer Res 2006;66:10497-10504.

40. Pevsner-Fischer M, Morad V, Cohen-Sfady M, Rousso-Noori L, ZaninZhorov A, Cohen S, et al. Toll-like receptors and their ligands control mesenchymal stem cell functions. Blood 2007;109:1422-1432.
4I. Poulain-Godefroy O, Froguel P. Preadipocyte response and impairment of differentiation in an inflammatory environment. Biochem Biophys Res Commun 2007;356:662-667.

42. Chung S, Lapoint K, Martinez K, Kennedy A, Boysen-Sandberg $M$, McIntosh MK. Preadipocytes mediate lipopolysaccharide-induced inflammation and insulin resistance in primary cultures of newly differentiated human adipocytes. Endocrinology 2006; 147:5340-535I. 